\title{
Designing and Engineering Interactive Computing Systems
}

\author{
MATHIAS FUNK, Eindhoven University of Technology, The Netherlands \\ RONG-HAO LIANG, Eindhoven University of Technology, The Netherlands \\ PHILIPPE PALANQUE, Université Paul Sabatier - Toulouse III, France \\ JUN HU, Eindhoven University of Technology, The Netherlands \\ PANOS MARKOPOULOS, Eindhoven University of Technology, The Netherlands
}

\begin{abstract}
This issue of the Proceedings of the ACM on Human-Computer Interaction features contributions in the intersection of human-computer interaction and software engineering, with further disciplines blending into a rich set of scientific works. 2021 is the first time the annual conference on Engineering Interactive Computing Systems (EICS) is hosted in the Netherlands and in the context of an Industrial Design department. We take this opportunity to focus on the relations and influence of the design discipline on the work of the EICS community. This resulted in a new set of topics for EICS, which were already partly reflected in the many submissions we received in three extensive review rounds throughout 2020 and the beginning of 2021. In this editorial we offer a perspective on what EICS is not yet, looking at the inclusion of and interplay with design as a related discipline.
\end{abstract}

CCS Concepts: • Human-centered computing $\rightarrow$ Interactive systems and tools; $\bullet$ Software and its engineering;

Additional Key Words and Phrases: Interactive systems, Model-based design of user interfaces, Model-driven Engineering, Software Engineering, User Interfaces, Design, Formal methods, Verification and Validation.

\section{ACM Reference Format:}

Mathias Funk, Rong-Hao Liang, Philippe Palanque, Jun Hu, and Panos Markopoulos. 2021. Designing and Engineering Interactive Computing Systems. Proc. ACM Hum.-Comput. Interact. 5, EICS, Article 193 (June 2021), 4 pages. https://doi.org/10.1145/3457140

\section{INTRODUCTION}

The EICS community researches, in the words of Calvary et al., "rich heterogeneity of this field can be expressed as deep ethnographies of information use in context, to experiments showing the effectiveness of interface designs, to the production of new technologies that push the limits of how we interact with computers, and much more" [2]. Also, "EICS focuses on models, languages, notations, methods, techniques, and tools that support the development life-cycle of interactive systems at any stage, from specification and requirements elicitation to validation" [1]. ACM EICS has historically grown from multiple strands reaching back to the 1990s, and exists now for more than ten years as an ACM conference. The PACMHCI track for EICS was established in 2017 and features impactful contributions with high visibility.

Authors' addresses: Mathias Funk, m.funk@tue.nl, Eindhoven University of Technology, Eindhoven, The Netherlands; Rong-Hao Liang, j.liang@tue.nl, Eindhoven University of Technology, Eindhoven, The Netherlands; Philippe Palanque, palanque@irit.fr, Université Paul Sabatier - Toulouse III, Toulouse, France; Jun Hu, j.hu@tue.nl, Eindhoven University of Technology, Eindhoven, The Netherlands; Panos Markopoulos, p.markopoulos@tue.nl, Eindhoven University of Technology, Eindhoven, The Netherlands.

Permission to make digital or hard copies of part or all of this work for personal or classroom use is granted without fee provided that copies are not made or distributed for profit or commercial advantage and that copies bear this notice and the full citation on the first page. Copyrights for third-party components of this work must be honored. For all other uses, contact the owner/author(s).

(C) 2021 Copyright held by the owner/author(s).

2573-0142/2021/6-ART193

https://doi.org/10.1145/3457140

Proc. ACM Hum.-Comput. Interact., Vol. 5, No. EICS, Article 193. Publication date: June 2021. 


\section{WHAT EICS IS NOT YET}

To respond to the earlier statements of "what EICS is" and "what EICS is not" by Lopez et al. [3] and later Bowen et al. [1], we offer a perspective of what EICS is not yet.

The field of EICS has a natural overlap with the design discipline, given the realization that "[b]uilding interactive systems is a multifaceted and challenging activity, involving a plethora of different actors and roles [...] where we continuously push the edge of what is possible [...], [to] help people cope with the ever-increasing complexity of work and life" [4]. Contemporary design research, be it influenced by interaction design, product design or service design, amongst others, targets and studies the complexity of humans interacting with computing systems in a context.

Design research shares with Software Engineering the direction to introduce novel, purpose-built artefacts and systems into a context. With HCI, design research shares the user-centered methods and approaches to generate new knowledge. What design researchers can add here is a sensitivity (1) for the particularity and diversity of contexts, that is, finding a balance between generalization and the given nuances and semantics of the field, (2) for the need to question additive technical solutions and providing ethnographic insights into long-term suitability, and (3) for aesthetics and other qualities that shape interactive systems in both design and engineering.

An area of particular interest in design research is the design (yes, and engineering) of tools and technology-mediated methods for design research and practice. That is, engineering interactive computing system for the purpose of design. This can be systems that help facilitate research, novel sensor systems and data collectors, and interactive data representations that integrate with contemporary remote research and partially ethnographic approaches to understand and design for complex, diverse and highly dynamic contexts: from the smart industry to smart mobility and energy grids to the mundane domestic Everyday.

\subsection{New topics for EICS}

A good way to introduce this perspective into the EICS community was to include it in a document that would be studied very seriously by prospective authors: the EICS 2021 Call for Papers. The list of proposed topics that contributing authors might use as a guide remained unchanged for several years, a perfect opportunity to changed it towards an extended list that casually embraces more designerly topics.

The most notable additions were (1) integrating engineering issues in the design process of interactive systems, (2) engineering design tools, and (3) supporting design in interactive development processes. The first caters to the needs to employ increasingly sophisticated remote design research and prototyping with a direct path to scaling-up. Consequently, engineering issues arise earlier in design processes and open new avenues for research bridging software engineering and design. The second new topic has a tradition in both EICS and also other ACM SIGCHI venues, yet the EICS community is particularly suited stakeholder with a focus on robust, reliable research products and systems. Third, takes a reverse perspective on what is covered in the first topic, aiming to increase the visibility and credibility of design work in development processes.

Further we respond to the interest of the community in working with emerging technologies highlighting this in one topic (formally "innovative applications"), and also extending the topic of engineering for user experience to the broader engineering interactive systems for specific properties (user experience, usability, safety, security, dependability, ...).

The final list of topics was clustered into three overarching clusters: (1) Modeling,Specification and Analysis, (2) Methods, Tools, and Processes, and (3) Applications and Integrations. Ultimately, we saw the need to introduce new experts to the collective of Associate Chairs, growing this committee to 28 accomplished researchers. 


\section{REPORTING}

Given the new selection of topics, we welcomed a variety of submission types, ranging from proof of concept, experiment and case study papers to comparative studies and formal analysis papers, to literature surveys and theoretical or formal methods papers.

In three rounds from July 2020 till April 2021, we received overall 62 valid submissions for all three rounds with ten accepted contributions in the first two rounds: in the first round which closed in July 2020, we received twelve valid submissions, of which four were accepted. The second round closed in October 2020, and we received 21 valid submissions, of which six were accepted. In the third and final round that closed in February 2021, we received 29 valid submissions, of which eleven were accepted with minor revisions ${ }^{1}$. Each valid submission was double-blindly reviewed by at least two external reviewers and one Associate Chair, with another Associate Chair providing a meta-review. Authors received notification with the possibility to submit a rebuttal that would then be taken into account in the PC meeting of the respective round.

When we look at the new set of topics clustered in three main contribution areas, "Application and Integration" and "Methods, Tools, and Process" received the majority of submissions across all rounds. We also note that twelve out of 62 valid submissions address design, designing or designers in their title directly. Two examples of a contribution responding to the new topic of design tools engineering are the works of Zhang et al. on automated, user-configurable, interactive agents for speech-tutoring and of Burny and Vanderdonckt on a workbench for conducting and reproducing experiments in GUI visual design. The work of Fernandez et al. on button designs for mid-air interaction in VR matches the new topic of "engineering interactive computing systems with emerging technologies", same as the works of Karolus et al. on a data-centric toolkit for EMG-based interface prototyping. The submissions also cover a wide variety of targeted domains and users, from industry and medical professionals, to developers and designers, to end-users. The most frequent contribution types are proof of concept and case study papers with aspects of formal analysis and experimental evidence.

\section{OUTLOOK}

While our above perspective on what EICS could evolve to is hopeful, this ultimately depends on the growing community and new authors trusting the community with their works. Judging from the submissions in the past three rounds, the new, broader selection of topics seems to resonate with the community. Yet, our vision for the future of the EICS community is constructive hypothesis and proposal.

In the next months, we look forward to high quality submissions. For researchers and practitioners in the intersection of software engineering, human-computer interaction and design, the EICS community plans to hold their next annual conference in Nice, France (see https://eics.acm.org/ 2020/) in 2022. This conference will provide many opportunities to share ideas with others from organizations around the world. More information about PACMHCI can be found at https://pacmhci. acm.org/ and about EICS at https://eics.acm.org.

\section{ACKNOWLEDGMENTS}

We thank the EICS Steering Committee, all involved Associate Chairs and external reviewers for their incredible work in shaping the selection of full paper contributions in this issue of PACMHCI.

\footnotetext{
${ }^{1}$ Provisional information available on the date of editorial submission, the final number of accepted papers in this round might vary.
} 


\section{REFERENCES}

[1] Judy Bowen, Marco Winckler, and Jean Vanderdonckt. 2020. A Glimpse into the Past, Present, and Future of Engineering Interactive Computing Systems. Proceedings of the ACM on Human-Computer Interaction 4, EICS (June 2020), 71:1-71:32. https://doi.org/10.1145/3394973

[2] Gaelle Calvary, Jeffrey Nichols, José Creissac Campos, Nuno Nunes, and Pedro Campos. 2017. Editorial Statement. Proceedings of the ACM on Human-Computer Interaction 1, EICS (June 2017), 1:1-1:2. https://doi.org/10.1145/3106386

[3] Víctor Manuel López Jaquero, Radu-Daniel Vatavu, Jose Ignacio Panach, Oscar Pastor, and Jean Vanderdonckt. 2019. A Newcomer's Guide to EICS, the Engineering Interactive Computing Systems Community. Proceedings of the ACM on Human-Computer Interaction 3, EICS (June 2019), 1:1-1:9. https://doi.org/10.1145/3300960

[4] Simone Stumpf and Jeffrey Nichols. 2018. Welcome Letter. Proceedings of the ACM on Human-Computer Interaction 2 , EICS (June 2018), 1:1. https://doi.org/10.1145/3179426 